\title{
MIMO radar signals with better correlation characteristics
}

\author{
Pavel Bezoušek ${ }^{1}$, Simeon Karamazov ${ }^{1 *}$
}

\begin{abstract}
MIMO radars employ multiple transmitting and receiving antennae. For each transmitting antenna, an independent and easily distinguishable signal is required, and appropriate filters must be used by the receiver. For this, the transmitted signals should have characteristics, enabling their effective separation. In this paper the correlation characteristics of selected signals are compared, and the appropriate signal coding is suggested. For differentiation, we address signals with basic linear or nonlinear frequency modulation (LFM or NLFM) multiplied by Gold, PRN, or frequency diversity (FD) codes. The analysis shows that better signal characteristics are achieved using the FD than the other codes. Using matched filters with filter length of 511, sidelobes and cross-correlations are suppressed by $40 \mathrm{~dB}$ with FD codes, while with the other codes only $20 \mathrm{~dB}$ was achieved. It was also proven, that the FD codes are more tolerant to the Doppler shift. On the other hand, the FD codes application leads to an extension of the overall transmitted signal bandwidth. This however, only represents a serious barrier for very broadband radar systems.
\end{abstract}

K e y w or d s: MIMO radar, autocorrelation function, time-frequency cross correlation, matched filter, frequency modulation, gold codes

\section{Introduction}

In recent years, MIMO (Multiple Input Multiple Output) radars have attracted significant attention, mainly due to their flexibility in space mapping and object resolution management (eg [1-7]. The MIMO radars are usually divided into two groups: one with collocated and the other with widely separated antennae. The term collocated means antennae separated by multiples of the wavelength and the widely separated antennae are separated by great multiples of space resolution cells. In the case of widely separated antennae, it is supposed that individual transmitter/receiver pairs observe their own target echoes, uncorrelated with the echoes of the other antenna pairs. Such radars take advantage of many uncorrelated paths leading to target detection enhancement at the cost of significant complications in data association and space scanning procedures. The radar with closely spaced antennae benefits from processing of correlated signals. This makes an adaptive generation of multiple variable antenna patterns possible. To avoid any uncertainty, in this paper we will deal only with radar systems with collocated antennae.

Apart from the basic MIMO radar theory, a number of publications discussed the generation of signals with optimum correlation characteristics, in the design of antenna characteristics or optimum signal processing [8-15]. Also critical comments (eg [16] are recorded. Some authors objected, that even the classical adaptive phased arrays could suppress interfering signals coming from multiple directions. The same is true for prevention of the signal transmission to unacceptable directions. To this point, it

could be stated, that the main MIMO system benefit is the extension of the digital adaptability on the transmitting antenna, providing extraordinary system management flexibility. Sometimes, objections are directed to a lower transmitting antenna gain of a MIMO radar, implying deterioration of its detection performance. This is a plausible criticism and several authors indicated and solved a tradeoff between detection and localization radar capabilities [17-19].

\section{MIMO radar model}

\subsection{Radar model description}

We consider a radar with $M$ transmitters and $N$ receivers with corresponding antenna elements, all situated along a line (x axis) according to Fig. 1. Targets are in the same plane as the antenna elements. Our analysis is thus restricted to the $2 \mathrm{D}$ scheme. The $3 \mathrm{D}$ situation is not addressed in this paper. The term $\Phi$ represents the angle between the target and the antenna axis in the mentioned plane. The consecutive element spacings in the transmitter and the receiver are denoted by $d_{T}$ and $d_{R}$ respectively, and the transmitter to receiver separation is labeled by $L$. The transmitted and received signals create column vectors $\mathbf{u}(t)$ and $\mathbf{s}(t)$ respectively

$$
\begin{aligned}
\mathbf{u}(t) & \equiv\left[u_{1}(t) u_{2}(t) \ldots u_{M}(t)\right]^{\top}, \\
\mathbf{s}(t) & \equiv\left[s_{1}(t) s_{2}(t) \ldots s_{N}(t)\right]^{\top} .
\end{aligned}
$$

\footnotetext{
${ }^{1}$ Faculty of Electrical Engineering and Informatics, University of Pardubice, Studentská 95, 53210 Pardubice, Czech Republic, * Corresponding author: simeon.karamazov@upce.cz
} 


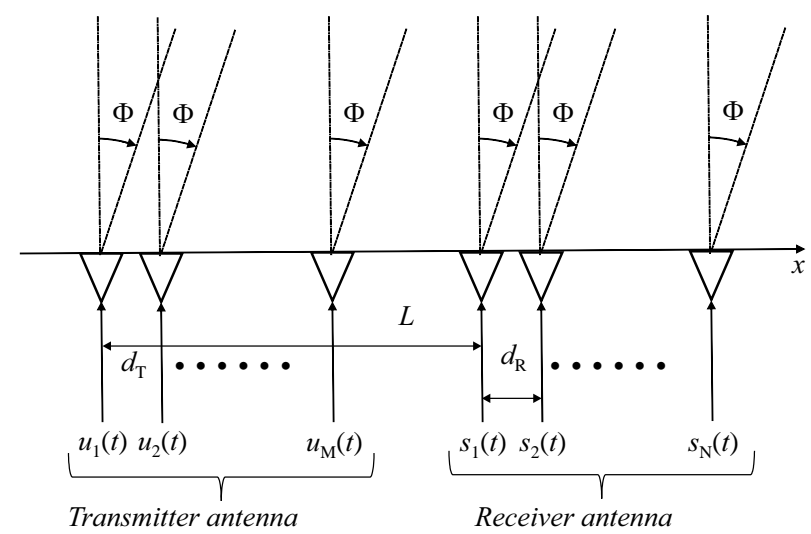

Fig. 1. Model of MIMO radar

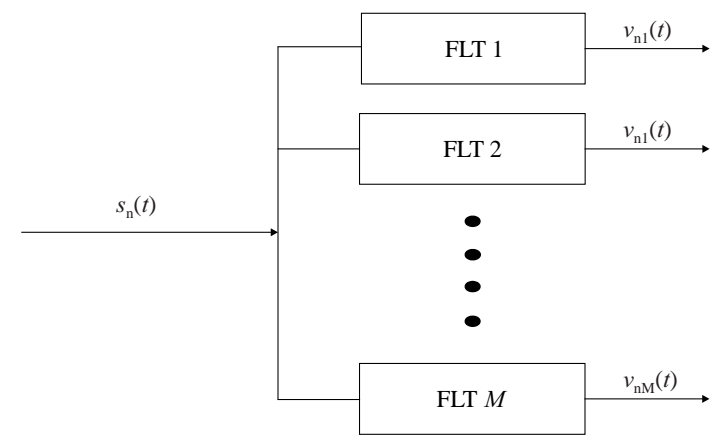

Fig. 2. Signal branching in the $n$-th receiver

Considering only one stationary point target, the two vectors are related by the following matrix equation

$$
\begin{gathered}
\mathbf{s}(t)=c_{T} \boldsymbol{A}(f) \mathbf{u}(t-\tau) \\
\boldsymbol{A}(f) \equiv\left[\begin{array}{c}
\mathbf{a}_{1}(f) \\
\vdots \\
\mathbf{a}_{N}(f)
\end{array}\right] \\
\mathbf{a}_{n}(f)=\left[a_{n 1}(f) a_{n 2}(f) \ldots a_{n M}(f)\right] \\
a_{n m}(f)=\exp \left[j 2 \pi\left(m \frac{d_{\mathrm{T}}}{\lambda}+n \frac{d_{\mathrm{R}}}{\lambda}\right) f\right] \exp \left[j 2 \pi \frac{L}{\lambda} f\right]
\end{gathered}
$$

where $\tau$ is a time delay of the reflected signal, $\boldsymbol{A}(f)$ is a steering matrix comprising steering vectors $\mathbf{a}_{n}(f)$ of this antenna array, spatial frequency $f=\sin \Phi$, and $\lambda$ is the wavelength. The complex constant $c_{\mathrm{T}}$ describes the target reflection.

At each receiver, the same bank of $M$ parallel filters processes the received signals (see Fig. 2), giving a $2 \mathrm{D}$ array of output signals

$$
\begin{aligned}
& \boldsymbol{V}(t)=\left[\begin{array}{ccc}
v_{11}(t) & \ldots & v_{1 M}(t) \\
\vdots & \ddots & \vdots \\
v_{N 1}(t) & \ldots & v_{N M}(t)
\end{array}\right] \\
& v_{n m}(t)=s_{n}(t) * q_{m}(t),
\end{aligned}
$$

where $v_{n m}(t)$ is an output signal in the $m$-th branch of the $n$-th receiver and $q_{m}(t)$ is the $m$-th branch filter response in the $n$-th receiver.

Combining (2) and (3) we get

$$
v_{n m}(t)=c_{T} \sum_{\mu=1}^{M} a_{n \nu}(f) r_{\mu m}(t-\tau),
$$

where $r_{\mu m}(t-\tau)=u_{\mu}(t-\tau) * q_{m}(t)$.

Output signals in all branches of all receivers are then linear combinations of delayed replicas of the set of $M \times N$ functions $r_{\mu m}(t)$.

In the case where matched filters are used, the filter responses are equal to

$$
q_{m}(t)=u_{m}^{*}\left(T_{\mathrm{D}}-t\right)
$$

where $T_{\mathrm{D}}$ is a fixed time delay. Then the functions $r_{n m}(t)$ are cross-correlation functions of the transmitted and received signals. Due to the Doppler effect, which plays a significant role in radars, the reflected signals are not only time-shifted but also frequency-shifted replicas. The signal processing then continues with another DFT through the individual pulse repetition intervals, leading to crossambiguity functions [20] of the transmitted/received signals. It is essential to transmit orthogonal signals to separate the individual transmitter/receiver paths.

The output signals from branching filters $v_{n m}(t)$, scattered by the same target are correlated. Then it is possible to digitally create joint transmitter/receiver antenna diagrams with $M \times N$ virtual antenna elements by linear combinations of these outputs (see eg [8]). It was also shown ( $e g[17])$ that it is possible to combine these $M$ transmitted signals at individual transmitting antennae to achieve the desirable transmit antenna diagram. This provides more degrees of freedom to radar designers.

Nowadays, MIMO technology starts to be employed for instance in automotive radars, where several producers offer $\mathrm{HW}$ platforms with more transmitters and receivers $(e g[21,22])$. The basic modulation is preferably LFM, and for diversifying, mostly PRN/BPSK modulations are used. But in many cases the frequency diversification (FD) of the transmitted signals would better fit the requirements. For example, long or middle range ACC (Automatic Cruise Control) radars use the basic modulation with the bandwidth of $1 \mathrm{GHz}$ or less. Perhaps even more fruitful may be application of the FD in Anti-Drone radars and generally in radars using the Track Before Detect (TBD) technology, which searches for a target immersed in a huge clutter. This paper points out the benefits of FD over the Gold or PRN codes application in such situations. 

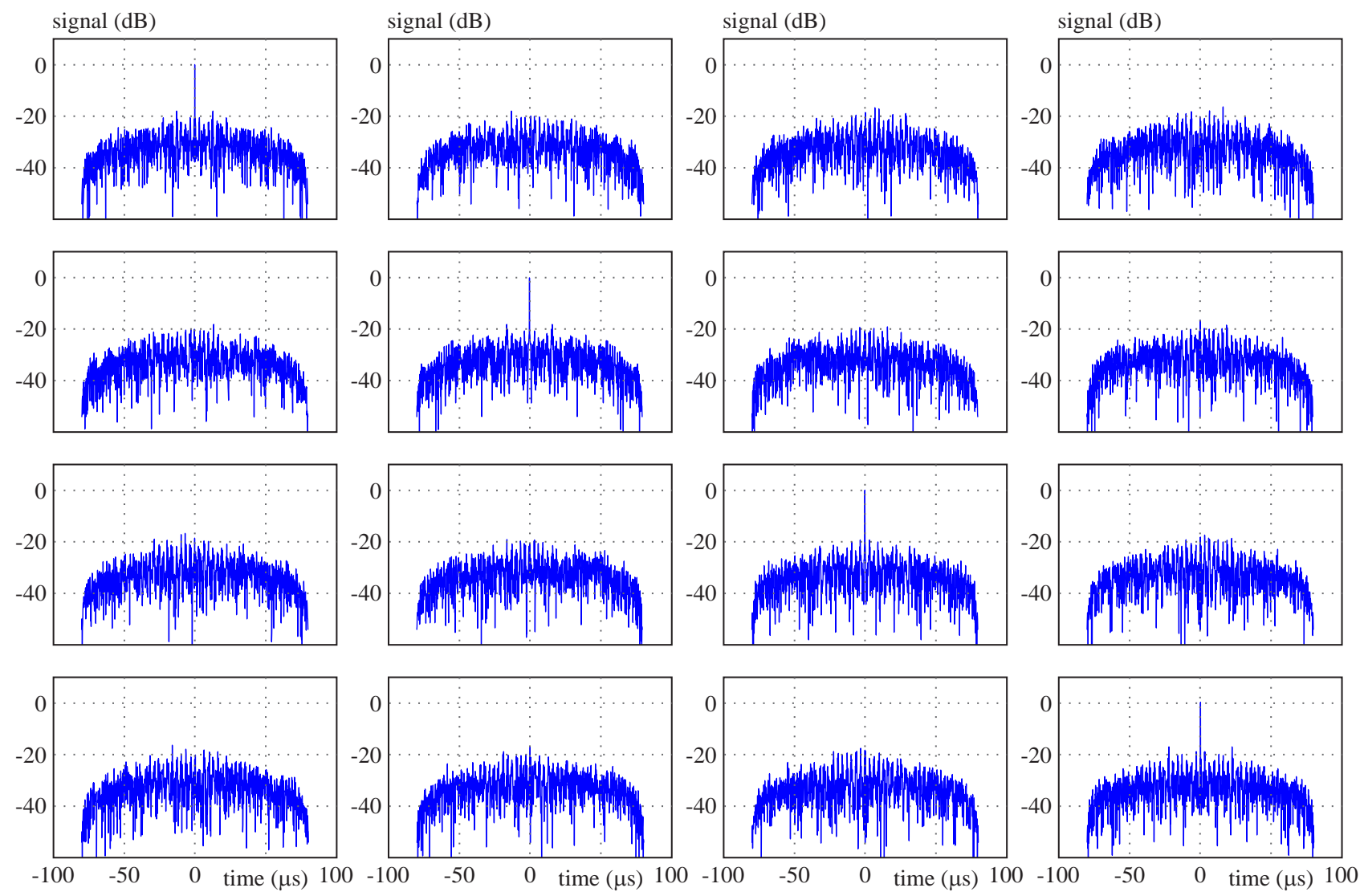

Fig. 3. Cross-correlations $r_{\mu m}(t)$. Transmitted signals LFM multiplied by Gold codes, $K=511$

\subsection{Waveform selection}

Radar signal modulations are subjects of some general constraints. For instance, to prevent signal distortion in high power amplifiers, constant-modulus modulations are usually preferred in radars. Further limitation comes from the necessity to handle Doppler shifted signals. Therefore, frequency modulations are preferred over direct pseudorandom noise code modulated PSK. In the MIMO radar, to generate $M$ orthogonal signals $u_{m}(t)$ for the separate $M$ transmitter signals, $M$ constant-modulus orthogonal code modulations $\left\{C_{m}(t)\right\}$ are needed

$$
u_{m}(t)=C_{m}(t) \cdot u_{0}(t)
$$

where: $u_{0}(t)$ is the basic FM with unit energy ie $\left\{C_{m}(t)\right\}$ is a set of orthogonal codes $C_{m}(t)= \pm 1$.

If the matched filters are used, then for the output signals $r_{\mu} m(t)$ at the output pulse center $t_{0}=\tau+T_{\mathrm{D}}$ according to (4) and (5) we have

$r_{\mu m}\left(t_{0}\right)=u_{\mu}\left(t_{0}\right) \cdot u_{m}^{*}\left(T_{\mathrm{D}}-t_{0}\right)=\int_{0}^{T_{\mathrm{D}}} u_{\mu}(t) u_{m}^{*}(t) \mathrm{d} t=$

$\int_{0}^{T_{\mathrm{D}}} u_{0}(t) u_{0}^{*}(t) C_{\mu}(t) C_{m}(t) \mathrm{d} t=\left|u_{0}\right|^{2} \int_{0}^{T_{\mathrm{D}}} C_{\mu}(t) C_{m}(t) \mathrm{d} t=\delta_{\mu m}$

$\left|u_{0}\right|^{2} \equiv\left|u_{0}(t)\right|^{2}=1$ (constant-modulus modulation), where, $\delta_{\mu m}=1$ for $\mu=m$ and $\delta_{\mu m}=0$ for $\mu \neq m$.
It means the transmitted signals are orthogonal in the time interval of $\left\langle 0, T_{\mathrm{D}}\right\rangle$.

The full output signal $r_{\mu m}(t)$ is equal to the crosscorrelation function of the transmitted signals $u_{\mu}(t)$ and $u_{m}(t)$. Its energy, appearing in significant indicators of signals usability in specific applications, can be expressed by its spectrum in the following way

$$
\begin{array}{r}
\varepsilon_{\mu m}=\int_{0}^{2 T_{\mathrm{P}}}\left|r_{\mu m}(t)\right|^{2} \mathrm{~d} t=\frac{1}{2 \pi} \int_{-\infty}^{\infty}\left|S_{\mu}^{*}(\omega) S_{m}(\omega)\right|^{2} \mathrm{~d} \omega \\
=\frac{1}{2 \pi} \int_{-\infty}^{\infty}\left|S_{\mu}(\omega)\right|^{2}\left|S_{m}(\omega)\right|^{2} \mathrm{~d} \omega
\end{array}
$$

where, $S_{m}(\omega)$ is the transmitted signal $u_{m}(t)$ is spectral density, and $\epsilon_{\mu m}$ is the energy of the output signal $r_{\mu m}(t)$.

At the output of the $m$-th branch filter all $r_{\mu m}(t)$, $\mu=1, \ldots M$ signals are present. The useful information is carried only by the main lobe (peak) of the $r_{m m}(t)$ signal, the rest act as interfering background. It consists of $r_{m m}(t)$ sidelobes and of cross-talks $r \mu \mathrm{m}(\mathrm{t})$, withall $\mu$ but $\mu=m$. From (8), it is evident, that to minimize the cross-talk energies $\varepsilon_{\mu m}$, the signals $u_{\mu}(t), u_{m}(t)$ with disjunctive spectra $\left|S_{\mu}(\omega)\right|^{2}$ and $\left|S_{m}(\omega)\right|^{2}$ are beneficiaries.

In this paper, we use two basic frequency modulations,

7) ie the linear frequency modulation (LFM) and the nonlinear one (NLFM) with a Taylor-shaped spectrum. In the role of overlay modulation, we compare Gold codes, PRN codes and frequency diversity (FD) modulations. 

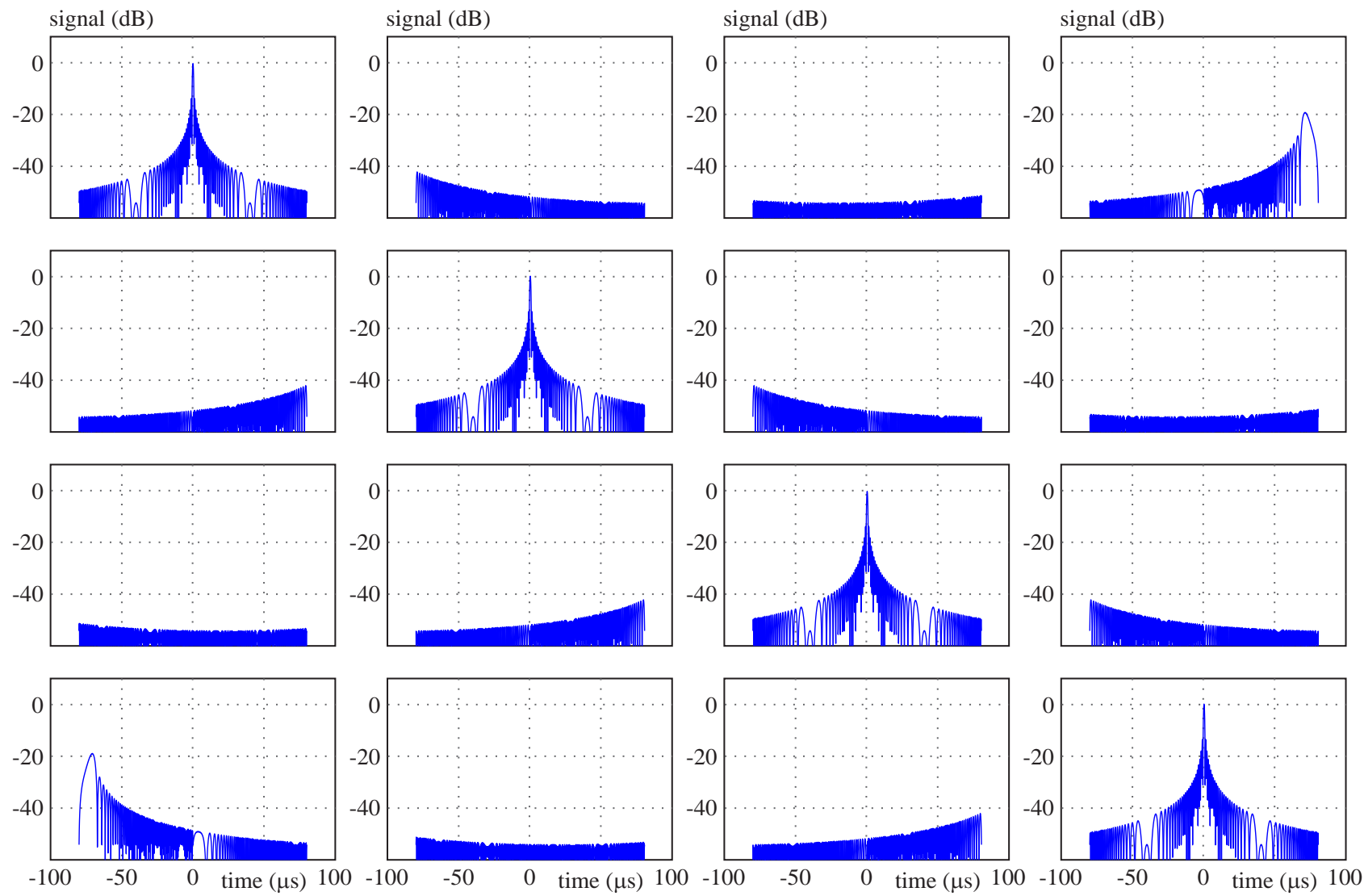

Fig. 4. Cross-correlations $r_{\mu m}(t)$. Transmitted signals $L F M$ multiplied by $F D$ codes, $K=511, k_{f}=1.2$

The basic modulations considered in this paper are internally frequency modulated pulses with the bandwidth of $B=1.25 \mathrm{MHz}$ and with the time-bandwidth product of $B \cdot T_{\mathrm{D}}=100$. The number of samples $K$ in the pulse is adapted to the bandwidth $B W$ of the whole signal set $u_{m}(t), m=1, \ldots M$, and to the nearest Gold code length with good cross-correlation characteristics. In further analysis we will deal with complex envelopes of the signals. In this representation, the FD modulations are performed by multiplication of the basic FM $u_{0}(t)$ by complex exponentials

$$
\begin{aligned}
& C_{m}(t)=\exp \left(j m 2 \pi k_{f} B t\right), \\
& B W=\left[(M-1) k_{f}+1\right] B,
\end{aligned}
$$

where, $k_{f}$ is an adjacent channels separation coefficient, and $B W$ is the overall bandwidth of the $M$ transmitted signals.

\section{Analysis of simulation result}

\subsection{Application of Gold and PRN codes}

In the following demonstration, we use $M=4$ transmitted signals, created from frequency-modulated pulses (LFM or NLFM - see section 2.2) multiplied by sets of orthogonal codes; ie Gold codes, PRN codes and FD codes.
We will analyze output signals in four branches of one of the receiver elements, and therefore we will deal with sets of $4 \times 4$ filter responses $r_{\mu m}(t)$ for each set of the transmitted signals. The sampling frequency is adapted to the maximum bandwidth of the analyzed transmitted signals. In our case, we need to adapt sampling to the overall bandwidth $B W$ of the frequency diversity code. The number $K$ of signal samples in a pulse should then satisfy $K \geq B W T_{\mathrm{D}}$. It is true, that the number of Gold and PRN separation code elements could be even lower, but such modifications do not improve the resulting parameters. That is why we use these codes of the length $K$.

Our results for the transmitted signals modulated by the LFM basic modulation and Gold codes are displayed in Fig. 3. The diagonal plots correspond to the autocorrelation functions and the lateral ones to the crosscorrelation functions of the transmitted signals. We can see, that the sidelobes as well as the cross-correlations attain the level of about $-20 \mathrm{~dB}$ of the autocorrelation peak amplitude. Such a high background level represents a definite drawback in specific radar applications, where a point target is surrounded by space clutter or a continuously spread matter (like rain, clouds, soil, etc). Then the background reflections of other resolution cells are added to the reflection of the actual cell. This increases target masking in clutter or distorts the continuum characteristics measurement. In such situations the integrated background energy in the $m$-th branch $E_{\mathrm{B} m}$ should be 

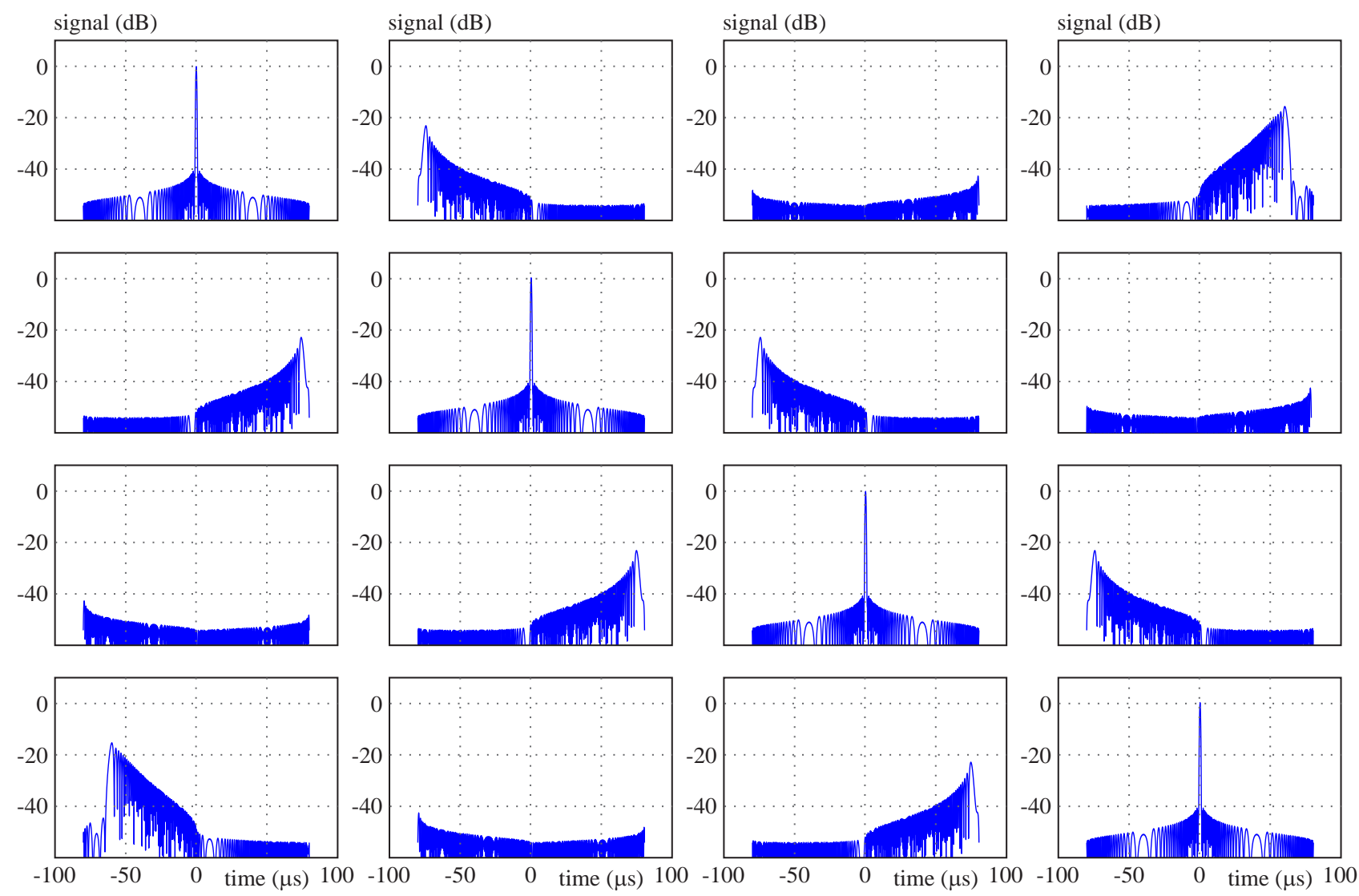

Fig. 5. Cross-correlations $r_{\mu m}(t)$. Transmitted signals NLFM multiplied by FD codes, $K=511, k_{\mathrm{f}}=1.2$

substantially smaller than the energy $E_{0 m}$ in the main lobe of the $m$-th transmitted signal autocorrelation function

$$
\begin{aligned}
E_{B m} & =\sum_{=1}^{M} \varepsilon_{\mu m}-E_{0 m}<<E_{0 m}, \\
R_{m} & =\frac{E_{B} m}{E_{0} m}=\frac{\sum_{\mu=1}^{M} \varepsilon_{\mu m}-E_{0} m}{E_{0} m} \ll 1,
\end{aligned}
$$

where $E_{0 m}$ is a main lobe energy of the $r_{m m}(t), E_{B m}$ is the total energy of all background signals in the $m$ branch, and $R_{m}$ is the ratio of the background energy to the main lobe energy in the $m$-th branch.

In the case shown in Fig. 3, the relevant quantities are $R_{m}=3.86$, which seems unsatisfactory in many cases. In the case of a greater number $M$ of independent transmitted signals, the impact of the background would be even worse. Usually, to minimize only autocorrelation function side-lobes, a nonlinear FM is used instead of the linear one. However, the ratio of the background to the main lobe energy for the Taylor shaped basic NLFM multiplied by the same set of the Gold codes has not changed substantially. Here we get: $R_{m}=3.84$. The results for the PRN codes used to diversify the transmitted signals are practically the same and the background to the main lobe energy ratio have a similar level. In this case, the result of energy computation is $R_{m}=3.98$. Even if the Gold or PRN code lengths are reduced, no improvement in this ratio is achieved. This is due to relatively bad cross-correlations of the used codes.

\subsection{Frequency shift codes}

It is clear, that this problem could be better solved, using time multiplexing of the transmitted signals. It eliminates simultaneous receptions of reflections of all transmitted signals. The signals could then be optimized in respect to their autocorrelation sidelobes only. However, this approach is not acceptable in most cases due to a large extension of the update period.

Another solution is to use frequency multiplexing. The corresponding correlations are shown in Figs. 4 and 5 . Here the FD codes (9) are used to diversify the basic linear and nonlinear frequency modulations. We find out that $R_{m}=0.11$ in the case of LFM multiplied by FD codes, and $R_{m}=0.099$ in the NLFM case. This improvement when compared to Gold and PRN codes could be seen at the first glance in the mentioned figures. The small difference in the energy ratios between LFM and NLFM is not substantial. However, if we study the sidelobes and the cross-correlation contributions separately, we can see, that at the LFM contribution of sidelobes is greater than that caused by cross-correlations. In the case of the NLFM, the situation is reversed. It indicates, 
that if we further extend the signal frequency separations (increasing the coefficient $k_{\mathrm{f}}$ ), the background selfinterference decreases much faster in case of NLFM then in the LFM case. When $k_{\mathrm{f}}=1.4$ is selected, the $R_{m}$ ratios for LFM and NLFM basic modulations give values of 0.1 and 0.033 respectively.

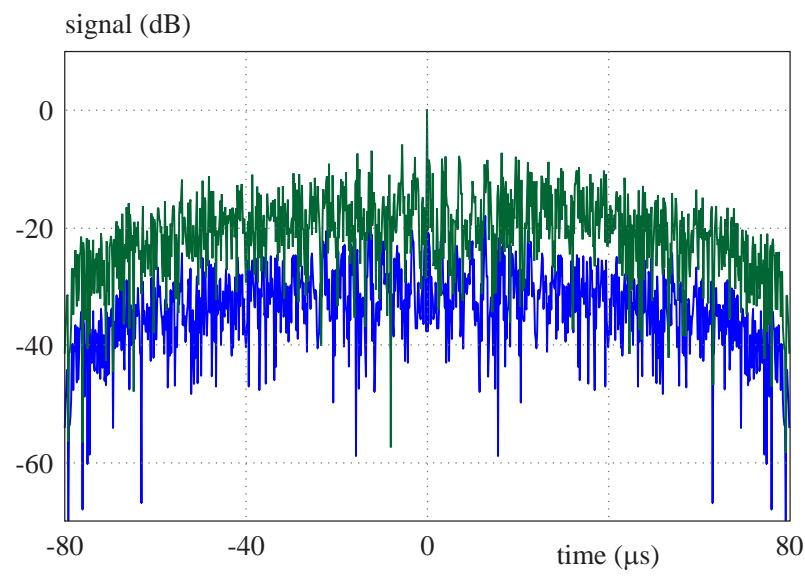

Fig. 6. Doppler shift impact on response in the corresponding branch - blue: $f_{d}=0$, green: $f_{d}=10 \mathrm{kHz}$. Transmitted signal is multiplied by Gold code

\subsection{Impact of the Doppler effect on signal correlation}

The Doppler shift of the received signal, due to the target or clutter motion, generally deteriorates the signal correlation characteristics. Obviously, the radar designers aim is to minimize this impact. It is well known that frequency modulated waveforms are less sensitive to the Doppler shift then the code modulations. The effect is completely described by cross-ambiguity functions (eg [20]) $C A F\left(\tau, f_{\mathrm{d}}\right)$, being functions of both time delay $\tau$ and the Doppler shift $f_{\mathrm{d}}$. For simplicitys sake, only the effects of a frequency shift of $10 \mathrm{kHz}$ on the output of the corresponding filter ( $i e$ the $r_{11}$ only) is shown in Fig. 6, in the case when Gold codes are used for signal diversification. Besides an unchanged tiny peak at the plot center, we can see side-lobes, considerably raised in the presence of the Doppler shift. The responses at non-corresponding branches (not shown here) are similar except for the central peak. The side-lobes and cross-correlation levels increase by $10 \mathrm{~dB}$ due to the $10 \mathrm{kHz}$ Doppler shift. On the other hand, the impact on the signal with FD codes is negligible and therefore it is not presented here.

\section{Conclusion}

In this paper, application of Gold, PRN and frequency diversity code modulations in MIMO radar are compared. The frequency diversity (FD) codes show lower sidelobes and cross-correlations than the other ones. FD codes facilitate good radar operation even in dense clutter. They guarantee high measurement quality of radars and better mapping of a distributed continuum, like in weather radars, ground mapping radars etc. Moreover, this modulation is much less sensitive to the Doppler shift, caused by moving reflection objects. In the Gold code modulated signals the Doppler effect significantly affects the radar background self-interference. In aircraft-tracking land-based radars, the immunity to the Doppler sidelobe (and cross-correlation) deterioration need not to be too significant, because the massive reflections usually show zero or small Doppler shift. On the other hand, the airborn and weather radars will certainly benefit from this quality.

However, the use of the frequency diversity codes leads to bandwidth widening, which may be a great drawback of this method. This is mainly in the case of broadband basic signals, like in FMCW systems with $\mathrm{GHz}$ bandwidths.

Looking to Figs. 4 and 5, we realize that the shape of the cross-correlations are not optimal and that there is some potential for future additional optimization of branching filters. The same could be anticipated for the Doppler Effect impact on the signal separation in the receiver.

\section{REFERENCES}

[1] J. Li, P. Stoica, (Ed.) MIMO Radar Signal Processing, John Wiley \& Sons Inc, 2009.

[2] B. J. Donnet and I. D. Longstaff, "MIMO Radar, Techniques and Opportunities", Proc. 3rd European Radar Conf., Manchester, UK, 2006, pp. 112-115.

[3] D. W. Bliss and K. W. Forsythe, "Multiple-Input Multiple Output (MIMO) Radar and Imaging: Degrees of Freedom and Resolution", Proc. 23th Asilomar Conf. Signals, Systems and Computers, vol. 1, pp. 54-59, Pacific Groove, CA, USA, November 2003.

[4] L. Xu, J. Li and P. Stoica, "Radar Imaging via Adaptive MIMO Techniques", Proc. 14th European Signal Processing Conf. (EUSIPCO`06), Florence, Italy, September 2006.

[5] N. H. Lehmann, A. M. Haimovich, R. S. Blum, and L. Cimini, "High Resolution Capabilities of MIMO Radar", Proc. 40th Asilomar Conf. Signals, Systems and Computers, pp. 25-30, Pacific Groove, CA, USA, November 2006.

[6] E. Fishler, A. Haimovich, R. S. Blum et al, "MIMO Radar: An Idea whose Time Has Come", Proc. IEEE Radar Conf. pp. 71-77, Philadelphia, USA, 2004.

[7] S. Haykin, "Cognitive Radar: A Way of the Future", IEEE Signal Procesing Magazine, vol. 23, no. 1, pp. 30-40, 2006.

[8] G. V. K. Sharma and K. R. Rajeswari, "Fast Implementation of Transmit Beamforming for Collocated Radar", Radiengineering, vol. 24, no. 1, pp. 171-177, 2015.

[9] G. Rossetti and S. Lambotharan, "Waveform Otimization Techniques for Bi-Static Cognitive radars", (CSPA2016), IEEE 12th International Colloquium on Signla Processing and its Application, pp. 115-118, Melaka, Malaysia, March 2016.

[10] J. Li, P. Stoica, and Y. Xie, "On Probing Signal Design for MIMO Radar", IEEE Transaction. on Signal Processing, vol. 55, no. 8, pp. 4151-4161, 2007.

[11] N. Lehmann, E. Fishler, A. Haimovich et al, "Evaluation of Transmit Diversity MIMO-Radar Direction Finding", IEEE Transaction on Signal Processing, vol. 55, no. 5, pp. 2215-2225, 2007.

[12] P. F. Sammartino, F. J. Guasch, and D. Tarchi, "Efficient Signal Processing MIMO Radars", Proc. IEEE European Radar Conf., EURAD 2011, pp. 13-16, Manchester, UK, 2011.

[13] K. Shadi and F. Behnia, "Transmit Beampattern Synthesis using Eigenvalue Decomposition MIMO Radar", Proc. 8th Int. Conf. on Information, Communication and Signal Processing, Singapore, December 2011. 
[14] R. A. Romero and N. A. Goodman, "Adaptive Beemsteering for Search-and-Track Aplication with Cognitive Radar Network", Proc. IEEE Nat. Radar Conf., pp. 1091-1095, Kansas City, Missouri, USA, 2011.

[15] D. S. Fuhrmann and G. San Antonio, "Transmit Beamforming for MIMO Radar Systems using Signal Cross-Correlation", IEEE Transaction on Aerospace Electronic Systems, vol. 44, no. 1, pp. 171-186, 2008.

[16] E. Brookner, "MIMO Radars and their Conventional Equivalents", CIE Radar, Guangzhou, China, 2016.

[17] M. Nazari Majd, M. Radmard, M. M. Chigarha, M. H. Bastani, and M. M. Nayebi, "Detection-Localisation Tradeoff MIMO Radars", Radioenegineering, vol. 26, no. 2, pp. 581-587, 2017.

[18] E. Fishler, A. Haimovich, R. S. Blum et al, "Spatial Diversity Radars-Models and Detection Performance", IEEE Transaction and Signal Processing, vol. 54, no. 3, pp. 823-838, 2006.

[19] L. Zheng and D. Tse, "Diversity and Multiplexing: A Fundamental Tradeoff Multiple-Antenna Channels", Transactions on Information Theory, vol. 49, no. 5, pp. 1073-1096, 2003.

[20] G. San Antonio, D. S. Fuhrmann, and F. Robey, "MIMO Radar Ambiguity Functions", IEEE Journal of Selected Topics Signal Processing, vol. 1, no. 1, pp. 167-177, 2007.

[21] Texas Instruments: AWR1843 Single-Chip 77 to 79 GHz FMCW Radar Sensor TI Datasheet, http://www.ti.com/lit/ds/swrs222a /swrs222a.pdf.
[22] V. Giannini, M. Hegdem and C. Davis, "Digital Code Modulation MIMO Radar Improves Automotive Safety", Microwave Journal, vol. 62, no. 8, pp. 106-118, 2019.

Received 19 March 2020

Pavel Bezoušek (prof, MSc, $\mathrm{PhD}$ ) was born in Ostrava, Czechoslovakia in 1943. He received his MS degree from the Czech Technical University in Prague in 1966 and the PhD degree from the same university in 1980 . He was with the Radio Research Institute of the Tesla Pardubice from 1966 till 1994, where he was engaged in microwave circuits and systems design. Since then he is with the University of Pardubice, now at the Faculty of Electrical Engineering and Informatics as a full professor, currently concerning with the radar systems design.

Simon Karamazov (prof, Dr Ing) was born in Pardubice, Czechoslovakia in 1963. He received his Ing. degree from the Czech Technical University in Prague in 1987 and his Dr degree from University of Pardubice in 1994 where he passed his doctoral study in solid state physics and semiconductors. Since 1991 he has been working at the University of Pardubice, now at the Faculty of Electrical Engineering and Informatics as a full professor of Department of Mathematics and Physics. 